\section{Directrices para el uso de antirretrovíricos en la infección por VIH en pacientes pediátricos ${ }^{1}$}

Palabras clave: antirretrovíricos, SIDA, VIH, infección pediátrica.
En 1993, el Grupo de Trabajo sobre el Tratamiento Antirretrovírico y el Tratamiento Médico de los Niños Infectados por VIH (en adelante, Grupo de Trabajo) concluyó que el tratamiento antirretrovírico está indicado en todo niño con un diagnóstico definitivo de infección por el virus de la inmunodeficiencia humana (VIH), acompañado de signos de inmunodeficiencia considerable o de síntomas asociados a la infección por VIH. Entonces se recomendó el tratamiento con zidovudina. En cambio, no se recomendó el tratamiento para los niños asintomáticos o con síntomas mínimos y estado inmunitario normal. Desde entonces han tenido lugar grandes adelantos que han producido importantes cambios en el tratamiento y la monitorización de la infección en los Estados Unidos de América (EE UU).

Aunque la patogénesis de la infección por VIH y los principios virológicos e inmunológicos en los que se basa el tratamiento antirretrovírico son iguales en todas las personas infectadas, en los lactantes, niños y adolescentes hay aspectos peculiares a tener en cuenta. En los niños, la mayoría de las infecciones por VIH son adquiridas en el período perinatal y la mayoría de las transmisiones perinatales ocurren durante el parto o cerca del mismo, lo cual señala la posibilidad de iniciar el tratamiento durante la fase inicial de la infección (infección primaria). Como la infección perinatal ocurre durante el desarrollo del sistema inmunitario del niño, tanto sus manifestaciones clínicas como la evolución de los marcadores virológicos e inmunológicos son distintas de las observadas en los adultos. Además, la farmacocinética de los antirretrovíricos también sufre grandes cambios entre el período neonatal y la edad adulta.

El Grupo de Trabajo se volvió a reunir en julio de 1997 para elaborar nuevas directrices que fueron publicadas en 1998 y que se actualizan en el presente informe. Estas directrices tratan de ser flexibles, no pretenden suplantar el juicio clínico de los profesionales sanitarios con amplia experiencia en este campo y serán revisadas periódicamente, a medida que se vaya obteniendo nueva información. En su formulación se tuvieron en cuenta los siguientes conceptos:

- La identificación de las mujeres infectadas antes del embarazo o a lo largo del mismo es crucial 
para poder proporcionar el mejor tratamiento, tanto a la madre como al niño, y para prevenir la transmisión perinatal.

- La participación de las embarazadas infectadas, de los recién nacidos expuestos y de los lactantes, niños y adolescentes infectados en ensayos clínicos constituye la mejor forma de identificar tratamientos eficaces y seguros.

- Las compañías farmacéuticas y el gobierno federal deberían colaborar para asegurar que las formulaciones adecuadas para lactantes y niños estén disponibles desde el mismo momento en que también lo estén para los adultos.

- Aunque se puede extrapolar alguna información sobre la eficacia de los antirretrovíricos a partir de los ensayos clínicos realizados en adultos, es necesario realizar estudios de este tipo en la población infantil.

- Todos los antirretrovíricos aprobados para el tratamiento de la infección por VIH se pueden usar en niños cuando estén indicados.

- Siempre que sea posible, el tratamiento de la infección por VIH en niños y adolescentes debería estar dirigido por un especialista en el tratamiento de la infección en esta población; si eso no fuera posible, se debería consultar regularmente con estos especialistas.

- El tratamiento eficaz requiere un abordaje multidisciplinario en el que participen médicos, enfermeras, trabajadores sociales, psicólogos, nutricionistas y otros profesionales.

- La determinación del número de copias del ARN del VIH y de los niveles de linfocitos T CD4 ${ }^{+}$es esencial para vigilar los efectos del tratamiento antirretrovírico y para modificarlo.

- Hay que tener en cuenta varios factores que influyen sobre el cumplimiento del tratamiento, como la disponibilidad de formulaciones pediátricas y su sabor, las repercusiones de las pautas terapéuticas sobre la calidad de vida, la capacidad de quienes cuidan del niño para administrarle regímenes terapéuticos complejos y las potenciales interacciones farmacológicas.

- La elección de los regímenes antirretrovíricos debería tomar en consideración factores asociados a una posible limitación de futuras opciones terapéuticas, como la resistencia a los antirretrovíricos. Las pruebas de resistencia pueden ser útiles, pero todavía no se ha establecido su valor en los niños y sus resultados deben ser interpretados por clínicos experimentados.

- La vigilancia del crecimiento y del desarrollo es un componente esencial de la atención a los niños infectados.

\section{ANTECEDENTES}

\section{Identificación de la exposición perinatal al VIH}

La identificación de las mujeres infectadas por VIH es fundamental, dado que permite:

- Administrarles tratamiento antirretrovírico y profilaxis frente a las infecciones oportunistas.

- Instaurar la quimioprofilaxis antirretrovírica con zidovudina, con el fin de reducir la transmisión perinatal del virus.

- Evitar la lactancia materna.

- Iniciar en todos los niños expuestos la profilaxis frente a la neumonía por Pneumocystis carinii a partir de las 4 a 6 semanas de vida.

- Diagnosticar rápidamente a los niños expuestos e instaurar el tratamiento de los infectados.

Cuando no se hayan efectuado pruebas de detección del VIH durante el embarazo, deberían realizarse en el período neonatal inmediato.

\section{Diagnóstico de la infección en el lactante}

El diagnóstico se puede establecer en la mayoría de los niños infectados al mes de vida, y en prácticamente todos a los 6 meses. La positividad de las pruebas virológicas indica una posible infección que se debe confirmar repitiendo la prueba cuanto antes en una segunda muestra. La prueba virológica preferida para establecer el diagnóstico en lactantes es la reacción en cadena de la polimerasa (RCP) para ADN. Las pruebas para detectar el ARN del VIH en el plasma también pueden ser útiles para el diagnóstico de la infección perinatal, y podrían ser incluso más sensibles que la RCP para ADN. El cultivo del VIH posee una sensibilidad similar a la de la RCP para ADN, pero es más complejo, más caro y tarda más ( 2 a 4 semanas) en proporcionar resultados. Las pruebas de detección del antígeno p24 son menos sensibles que otras pruebas virológicas y no se recomienda su empleo aislado en niños de menos de 1 mes, debido a la alta frecuencia de falsos positivos a estas edades.

Se recomienda la realización de la prueba inicial a las 48 h de vida porque cerca del $40 \%$ de los niños infectados ya pueden ser identificados en este momento. No se deberían utilizar muestras de sangre del cordón umbilical debido al riesgo de contaminación con sangre materna. Se considera que los niños con pruebas virológicas positivas en este momento han sufrido infección intrauterina y que 
aquellos en los que las pruebas se positivizan posteriormente han sido infectados durante el parto. En niños con pruebas negativas tras el parto se puede considerar su repetición a la edad de 14 días, puesto que la sensibilidad diagnóstica de las pruebas virológicas aumenta rápidamente en este período.

En los casos inicialmente negativos se deberían repetir las pruebas virológicas al mes o a los 2 meses de vida. Si siguen siendo negativas, hay que volver a repetirlas entre los 3 y los 6 meses de edad. Es razonable descartar la existencia de infección en niños con dos o más pruebas virológicas negativas, dos de las cuales hayan sido realizadas después del mes de vida, y una de estas después de los 4 meses. Siempre que no existan signos clínicos de infección, también es razonable suponer la ausencia de infección tras la obtención de dos o más pruebas negativas de IgG anti-VIH realizadas después de los 6 meses, con un intervalo de al menos un mes entre ellas. La infección se puede excluir definitivamente cuando la prueba de IgG anti-VIH sea negativa en ausencia de hipogammaglobulinemia a los 18 meses, las pruebas virológicas también sean negativas y no existan síntomas clínicos de infección por VIH.

\section{Vigilancia de la infección pediátrica por VIH}

Parámetros inmunológicos en niños. Al interpretar los recuentos de linfocitos $\mathrm{T} \mathrm{CD}^{+}$en niños hay que tener en cuenta la edad. En lactantes sanos, tanto el recuento absoluto como el porcentaje de estas células son considerablemente mayores que en adultos sanos y van disminuyendo lentamente hasta alcanzar los valores de los adultos hacia los 6 años. Aunque los recuentos absolutos que identifican determinado nivel de inmunodepresión cambian con la edad, no ocurre lo mismo con el porcentaje de estas células que define cada categoría inmunológica (cuadro 1). Por lo tanto, en los niños, los cambios del porcentaje de células $\mathrm{CD}^{+}$pueden ser mejores indicadores de la progresión de la en- fermedad que los recuentos absolutos. Como el estado inmunitario es fundamental para poder proporcionar la mejor atención a los lactantes y niños infectados, los recuentos y porcentajes de células $\mathrm{CD}^{+}$se deben determinar tan pronto como sea posible tras el diagnóstico virológico, y repetirse cada 3 meses. Los niños infectados con un perfil inmunofenotípico característico de defecto tímico (recuento de células $\mathrm{CD}^{+}<1900 / \mathrm{mm}^{3}$ y de células $\mathrm{CD}^{+}>850 / \mathrm{mm}^{3}$ ) durante los primeros 6 meses de vida tienen una progresión más rápida que los lactantes sin este perfil. Los valores de los linfocitos T $\mathrm{CD}^{+}$tienen considerables variaciones en un mismo paciente. Las enfermedades intercurrentes leves o las vacunaciones pueden producir disminuciones transitorias de los recuentos y porcentajes, por lo que sus determinaciones se deben efectuar cuando el paciente se encuentra clínicamente estable. Los tratamientos solo se deberían modificar debido a cambios de estos parámetros después de confirmarlos con al menos una segunda determinación separada por un intervalo de al menos 1 semana.

ARN del VIH en niños. La carga vírica en sangre periférica se puede determinar con pruebas cuantitativas del ARN del VIH. En los adultos, durante la infección primaria el número de copias del ARN del virus aumenta inicialmente hasta niveles muy elevados, pero coincidiendo con la aparición de la respuesta inmunitaria humoral y celular llegan a disminuir hasta en 2 a 3 órdenes de magnitud $\left(\log _{10}\right)$ para alcanzar un nivel estable más bajo (el llamado "punto de equilibrio" virológico) unos 6 a 12 meses después de la infección aguda, cuando se establece el equilibrio entre la producción y la eliminación del virus. Varios estudios realizados en adultos han indicado que los pacientes con valores del "punto de equilibrio" más bajos tienen una progresión más lenta y mejor supervivencia que aquellos con valores más elevados. Esto ha llevado a la elaboración de recomendaciones para iniciar o cambiar el tratamiento antirretrovírico en función del número de

CUADRO 1. Sistema de clasificación de la infección pediátrica por VIH (revisión de 1994): categorías inmunológicas basadas en los recuentos absolutos y los porcentajes de linfocitos $\mathrm{T} \mathrm{CD4} 4^{+}$en función de la edad

\begin{tabular}{|c|c|c|c|c|c|c|}
\hline \multirow[b]{2}{*}{ Categoría inmunológica } & \multicolumn{2}{|c|}{$<12$ meses } & \multicolumn{2}{|c|}{1 a 5 años } & \multicolumn{2}{|c|}{6 a 12 años } \\
\hline & No. $/ \mathrm{mm}^{3}$ & $\%$ & No. $/ \mathrm{mm}^{3}$ & $\%$ & No. $/ \mathrm{mm}^{3}$ & $\%$ \\
\hline 1: sin inmunodepresión & $\geq 1500$ & $\geq 25$ & $\geq 1000$ & $\geq 25$ & $\geq 500$ & $\geq 25$ \\
\hline 2: inmunodepresión moderada & 750 a 1499 & $15-24$ & 500 a 999 & $15-24$ & 200 a 499 & $15-24$ \\
\hline 3: inmunodepresión grave & $<750$ & $<15$ & $<500$ & $<15$ & $<200$ & $<15$ \\
\hline
\end{tabular}


copias del ARN del VIH, recomendaciones que también son aplicables a los adolescentes, en particular a los que han adquirido la infección recientemente y no en el período neonatal, y posiblemente a los niños de más de 3 años con infección perinatal.

La evolución del ARN del VIH en los niños con infección adquirida en el período neonatal es diferente de la observada en adultos. Los números elevados de copias persisten en los niños durante prolongados períodos de tiempo y tras el primer año de vida disminuyen lentamente a lo largo de varios años. Esta evolución probablemente refleje la menor eficiencia del sistema inmunitario en desarrollo a la hora de contener la replicación vírica y, posiblemente, también la existencia de un mayor número de células susceptibles al VIH.

Aunque las concentraciones de ARN del VIH presentan una considerable sobreposición en los lactantes con y sin progresión rápida de la enfermedad, las concentraciones elevadas (> 100000 copias $/ \mathrm{mL}$ ) en lactantes se han asociado a un alto riesgo de progresión de la enfermedad y muerte, sobre todo cuando el porcentaje de células $\mathrm{CD}^{+}$es $<15 \%$. En un estudio, el riesgo relativo de progresión de la enfermedad disminuyó un $54 \%$ con cada reducción de $1 \log _{10}$ en la concentración basal de ARN del VIH. Los datos obtenidos en niños mayores de 30 meses han sido similares a los de los adultos, con un considerable aumento del riesgo de progresión de la enfermedad cuando las cifras son superiores a 10000 a 20000 copias/mL. De cualquier forma, el valor predictivo de las concentraciones de ARN del VIH con respecto a la progresión de la enfermedad y a la muerte es tan solo moderado en pacientes individuales. También hay datos que indican que el uso combinado del número de copias del ARN del VIH y del porcentaje de células $\mathrm{CD}^{+}$ puede permitir una definición más exacta del pronóstico. En varios estudios realizados en adultos infectados se han obtenido resultados similares que avalan esta conclusión.

Consideraciones metodológicas sobre la interpretación y comparabilidad de las pruebas de detección del ARN del VIH. La mayoría de los datos publicados acerca del ARN del VIH en niños han sido obtenidos en muestras congeladas de plasma y suero. Como el almacenamiento de las muestras y el retraso de su procesamiento se asocia a una degradación del ARN del VIH, los datos publicados pueden no ser directamente comparables con los obtenidos en muestras procesadas inmediatamente. Por otra parte, diferentes estudios han utilizado distintas pruebas de detección del ARN del VIH, cada una de ellas con diferentes grados de sensibilidad.
Aunque los resultados de las diferentes pruebas están correlacionados entre sí, los valores absolutos obtenidos en la misma muestra con distintas pruebas pueden llegar a diferir en más de $0,3 \log _{10}$ (dos veces). Esto hace que se deba utilizar siempre la misma prueba para evaluar a un mismo paciente.

Sobre todo en niños pequeños, la elección de la prueba puede verse influenciada por la cantidad de sangre necesaria. La prueba de amplificación basada en la secuencia de ácidos nucleicos (NASBA) es la que requiere menor cantidad de plasma (100 $\mu \mathrm{L})$, seguida de la RCP cuantitativa Amplicor HIV-1 Monitor $(200 \mu \mathrm{L})$ y de la prueba de amplificación de la señal con ADN de cadena ramificada Quantiplex $(1 \mathrm{~mL})$.

Las concentraciones de ARN del VIH tienen una variabilidad biológica bien documentada. En adultos infectados clínicamente estables pueden variar en hasta $0,5 \log _{10}$ (tres veces), en un sentido o en otro, en un mismo día. Esta variabilidad puede ser todavía mayor en lactantes y niños pequeños. En niños con infección adquirida en el período perinatal, el número de copias disminuye lentamente durante los primeros años de vida, incluso en ausencia de tratamiento, aunque se mantiene persistentemente en niveles superiores a los de la mayoría de los adultos. Esta reducción es más rápida durante los primeros 12 a 24 meses de vida (media de 0,6 $\log _{10}$ al año), y sigue más lentamente (media de 0,3 $\log _{10}$ al año) hasta los 4 a 5 años. Esta variabilidad biológica debe ser tenida en cuenta al interpretar los cambios del número de copias del ARN del VIH en niños. Solo se deben considerar sustanciales desde el punto de vista clínico y biológico los cambios de más de $0,7 \log _{10}$ (cinco veces) en menores de 2 años y de más de $0,5 \log _{10}$ (tres veces) en los mayores de 2 años, y solo tras la repetición de las pruebas. Para reducir el impacto de esta variabilidad sobre la conducta clínica, se pueden obtener dos muestras basales y utilizar la media de los resultados de ambas para la comparación con las pruebas futuras. De lo anterior se concluye también que el tratamiento no se debe modificar debido a cambios en el número de copias del ARN del VIH, a no ser que hayan sido confirmados por una segunda determinación.

Problemas específicos del tratamiento antirretrovírico en adolescentes infectados por el VIH. $\mathrm{Al}$ tratamiento antirretrovírico de los adolescentes pospúberes infectados por vía sexual o por el consumo de drogas parenterales se le pueden aplicar las mismas normas que a los adultos, dado que estos pacientes suelen seguir una evolución más similar a la de los adultos que a la de los niños. En cambio, los adolescentes que adquirieron la infec- 
ción en el período perinatal, o en la primera infancia a través de productos sanguíneos, pueden presentar una evolución clínica distinta de la del grupo anterior. Como muchos adolescentes infectados por el VIH tienen actividad sexual, es imprescindible asesorarlos acerca de la anticoncepción y de la prevención de la transmisión sexual del virus.

Las dosis de las medicaciones antirretrovíricas y de los fármacos utilizados frente a las infecciones oportunistas deberían prescribirse en función de los estadios de Tanner, y no de la edad. Al inicio de la pubertad (estadios I y II de Tanner) se deben utilizar dosis pediátricas, mientras que al final de la misma (estadio $\mathrm{V}$ de Tanner) se deben utilizar las mismas dosis que en los adultos. Los jóvenes que se encuentren en la fase de crecimiento rápido (estadio III de Tanner en el sexo femenino y estadio IV en el sexo masculino) pueden ser tratados con las mismas dosis utilizadas en pacientes pediátricos o en adultos, pero deben ser vigilados estrechamente para controlar la eficacia y la seguridad del tratamiento.

Aunque los cambios fisiológicos de la pubertad, como el aumento de la grasa corporal en el sexo femenino y de la masa muscular en el sexo masculino, podrían afectar a la farmacocinética de las medicaciones utilizadas, no se han observado alteraciones clínicamente importantes con los inhibidores nucleosídicos de la transcriptasa inversa (INTI). La experiencia clínica con los inhibidores de la proteasa (IP) y los inhibidores no nucleosídicos de la transcriptasa inversa (INNTI) es más escasa.

Problemas específicos del cumplimiento del tratamiento antirretrovírico en niños y adolescentes infectados por el VIH. El incumplimiento del tratamiento y las concentraciones subterapéuticas de antirretrovíricos pueden potenciar la aparición de resistencia a los fármacos. Los datos existentes indican que la aparición de resistencia a un IP puede reducir la susceptibilidad a todos los demás fármacos de esta clase y, así, las futuras opciones terapéuticas. Lo mismo ocurre con los INNTI. Por consiguiente, al iniciar el tratamiento es necesario educar al niño o a quienes cuidan de él acerca de la importancia del cumplimiento del régimen terapéutico prescrito y seguir reforzando este mensaje en las consultas posteriores.

El cumplimiento del tratamiento es especialmente problemático en los niños. La toma de las medicaciones por los lactantes y los niños pequeños depende de terceros, por lo que resulta fundamental evaluar la capacidad de quienes cuidan de ellos para cumplir regímenes terapéuticos complejos. La administración oral a niños pequeños requiere for- mulaciones líquidas o que se puedan mezclar con la leche $\mathrm{u}$ otros alimentos y su mal sabor puede crear problemas. Por otra parte, la absorción de algunos antirretrovíricos puede verse afectada por las comidas. Además, en los niños y adolescentes existen muchos otros obstáculos al cumplimiento del tratamiento que hacen necesaria una completa evaluación de los problemas de adherencia antes de instaurar el tratamiento. Los pacientes deben ser observados frecuentemente para evaluar el cumplimiento del tratamiento, la tolerancia a los fármacos y la respuesta virológica. En algunas familias, antes de iniciar el tratamiento puede ser necesario resolver otros problemas prioritarios, como la seguridad del entorno físico, el alojamiento y la alimentación.

Los adolescentes también presentan problemas específicos, como la negación y el miedo a la infección por el VIH, la falta de información, la desconfianza en el sistema sanitario y la eficacia de la medicación, la baja autoestima, el estilo de vida desestructurado o caótico, la falta de apoyo familiar o el deseo de no diferenciarse de sus compañeros.

\section{RECOMENDACIONES TERAPÉUTICAS}

\section{Consideraciones generales}

Al considerar la posibilidad de iniciar el tratamiento antirretrovírico, los problemas relacionados con su cumplimiento adquieren especial importancia. El tratamiento tiene mayores probabilidades de éxito en pacientes que nunca han sido tratados y que, por lo tanto, tienen menos posibilidades de estar infectados por cepas resistentes a los antirretrovíricos. El incumplimiento del tratamiento y las concentraciones subterapéuticas de antirretrovíricos, sobre todo de los IP, pueden incrementar el riesgo de resistencia a los fármacos y la probabilidad de fracaso virológico. La participación de los niños y de quienes cuidan de ellos en la toma de decisiones es fundamental, sobre todo en situaciones en las que no hay datos definitivos sobre la eficacia del tratamiento.

Niños infectados con síntomas inmunológicos o clínicos. El tratamiento antirretrovírico les ha proporcionado importantes beneficios clínicos a los niños con síntomas clínicos o inmunológicos de infección por el VIH. Los ensayos clínicos iniciales sobre las monoterapias con zidovudina, didanosina, lamivudina o estavudina demostraron considerables mejorías desde el punto de vista del desarrollo neurológico, del crecimiento y del estado virológico o inmunológico. Estudios posteriores demostraron la superioridad de los tratamientos combinados en 
comparación con la monoterapia, no solo en el aspecto clínico, inmunológico y virológico, sino también en relación con la mortalidad.

Niños infectados asintomáticos. No está tan claro cuándo comenzar el tratamiento en niños asintomáticos con función inmunitaria normal, pues no hay datos procedentes de ensayos clínicos de fase III acerca de la eficacia del tratamiento antirretrovírico en este grupo de población. No obstante, al menos teóricamente, el inicio del tratamiento en el primer o segundo año de vida podría conservar la función inmunitaria, reducir la diseminación y la carga vírica y mejorar el desenlace clínico. De cualquier forma, la proporción de casos que alcanzan concentraciones de ARN del VIH inferiores al límite de detección con los tratamientos potentes es menor en los niños pequeños que en los niños mayores y en los adultos, en parte porque la respuesta virológica está relacionada con la carga vírica al comienzo del tratamiento y porque los niños pequeños tienen cargas víricas considerablemente mayores. Aun así, hay datos que indican que incluso una respuesta parcial puede proporcionar beneficios clínicos e inmunológicos.

Los potenciales problemas del tratamiento precoz incluyen el riesgo de efectos adversos a corto y a largo plazo. Además, si no se logra la supresión de la replicación del virus, aumenta la probabilidad de mutaciones productoras de resistencia, lo cual reduce la duración de los beneficios del tratamiento y puede reducir las futuras opciones terapéuticas.

\section{Cuándo comenzar el tratamiento}

Antes de iniciar el tratamiento antirretrivírico es fundamental aconsejar a los pacientes y a quienes cuidan de ellos acerca de la importancia del cumplimiento del régimen terapéutico prescrito. Previamente se deben identificar y resolver los problemas, aunque esto retrase el inicio del tratamiento. Además son necesarias consultas frecuentes para evaluar la respuesta virológica, la intolerancia a los fármacos, la resistencia a los antivíricos y la adhesión al tratamiento.

Niños infectados con síntomas clínicos e inmunológicos. Se recomienda el tratamiento antirretrovírico de todos los niños con síntomas clínicos de infección por VIH (esto es, en las categorías clínicas A, $\mathrm{B}$ o C) o signos de inmunodepresión (esto es, en las categorías inmunológicas 2 o 3) (cuadro 1), inde- pendientemente de la edad del niño o de su carga vírica. Los datos de los ensayos clínicos realizados tanto en adultos como en niños han demostrado que el tratamiento antirretrovírico de los pacientes sintomáticos reduce la progresión clínica e inmunológica de la enfermedad y la mortalidad.

Niños asintomáticos de menos de $\mathbf{1 2}$ meses. Lo ideal es comenzar el tratamiento antirretrovírico de todos los niños de menos de 12 meses tan pronto como se confirme el diagnóstico, independientemente del estado inmunitario y de la carga vírica. Los lactantes de menos de 1 año corren un alto riesgo de progresión de la enfermedad y los parámetros virológicos e inmunológicos tienen menor valor predictivo que en niños mayores con respecto a la progresión de la enfermedad. La información sobre la posología de los antirretrovíricos en lactantes de menos de 3 a 6 meses es escasa. Las funciones hepática y renal son inmaduras en el recién nacido y sufren importantes cambios durante los primeros meses de vida. Esto puede condicionar importantes diferencias de dosis entre los niños pequeños y los mayores.

Niños asintomáticos de más de $\mathbf{1 2}$ meses. Para estos niños, el Grupo de Trabajo ha definido dos abordajes generales. El primero consiste en instaurar el tratamiento de todos los niños, independientemente de su edad y estado clínico. De este modo se asegura el tratamiento de los niños infectados lo antes posible y antes del deterioro de la función inmunitaria. Los resultados de varios estudios prospectivos indican que la mayoría de los niños infectados presentan síntomas clínicos hacia el año de edad. Por otra parte, la mayoría de los niños asintomáticos mayores de 1 año tienen porcentajes de células $\mathrm{T} \mathrm{CD}^{+}<25 \%$, que indican inmunodepresión y justifican el tratamiento antirretrovírico.

El abordaje alternativo consiste en diferir el tratamiento de los niños con estado inmunitario normal en situaciones en las que el riesgo de progresión clínica de la enfermedad es bajo (esto es, baja carga vírica) y existen otros factores que favorecen el aplazamiento del inicio del tratamiento (por ejemplo, problemas de adhesión al tratamiento, seguridad y persistencia de la respuesta antirretrovírica). En tal caso hay que vigilar regularmente el estado virológico, inmunológico y clínico. Entre los factores a considerar en la decisión sobre cuándo iniciar el tratamiento se incluyen las concentraciones elevadas o crecientes del ARN del VIH, la reducción rápida de los recuentos o porcentajes de células $\mathrm{CD}^{+}$y su acercamiento a valores que indican in- 
munodepresión moderada (esto es, categoría inmunológica 2), y la aparición de síntomas clínicos.

La concentración de ARN del VIH indicativa de aumento del riesgo de progresión de la enfermedad no está bien definido en los niños pequeños. De cualquier forma, independientemente de la edad, cualquier niño con concentraciones de ARN del VIH $>100000$ copias/mL corre un alto riesgo de muerte $\mathrm{y}$ en estos casos se debe iniciar el tratamiento antirretrovírico independientemente del estado clínico o inmunológico. En niños mayores (> 30 meses), el riesgo de progresión de la enfermedad o muerte a los 2 años es muy bajo cuando la concentración de ARN del VIH es < 15000 copias/mL, pero por encima de esa cifra aumenta al 13\% o más. También se le debe ofrecer tratamiento antirretrovírico a todo niño cuya concentración de ARN del VIH aumente considerablemente (en más de 0,7 $\log _{10}$ en menores de 2 años y en más de $0,5 \log _{10}$ en mayores de 2 años), y esto con independencia de su carga vírica absoluta y de su estado clínico o inmunológico, y siempre que se hayan descartado otras explicaciones para dicho aumento, como las infecciones intercurrentes o las vacunaciones.

\section{Elección del tratamiento antirretrovírico inicial}

De acuerdo con los datos clínicos, inmunológicos y virológicos de los ensayos clínicos realizados en adultos y en niños, los regímenes antirretrovíricos se clasifican del siguiente modo: firmemente recomendados, recomendados como alternativa, ofrecidos en circunstancias especiales y no recomendados (cuadro 2).

Se recomienda el tratamiento combinado con al menos tres fármacos antiretrovíricos en todos los lactantes, niños y adolescentes. En comparación con la monoterapia, el tratamiento combinado reduce la progresión de la enfermedad y mejora la supervivencia, proporciona una respuesta virológica e inmunológica más intensa y sostenida, y retrasa la aparición de mutaciones de resistencia a los fármacos utilizados. La monoterapia ya no se recomienda. La excepción es la monoterapia con zidovudina en lactantes con estado virológico indeterminado durante las primeras 6 semanas de vida, con el fin de prevenir la transmisión perinatal del VIH. Una vez confirmada la infección, se debe cambiar la quimioprofilaxis con zidovudina por algún tratamiento combinado de los recomendados. En caso de que se decida aplazar el tratamiento, también se suspenderá la zidovudina, en espera de la decisión terapéutica.

Teóricamente, el régimen antirretrovírico inicial podría verse influenciado por el tratamiento que la madre haya recibido durante el embarazo, pero no hay datos clínicos que confirmen esta suposición. No obstante, cuando haya sospecha o confirmación de resistencia a un antirretrovírico en la madre de un lactante recién diagnosticado, se puede considerar la posibilidad de realizar pruebas de resistencia en el lactante con el fin de elegir el régimen terapéutico inicial.

El régimen antirretrovírico más recomendado para el tratamiento inicial consiste en la combina-

\section{CUADRO 2. Regímenes antirretrovíricos recomendados para el tratamiento inicial de la infección por VIH en niños}

\begin{tabular}{|c|c|}
\hline Recomendación & Tratamiento \\
\hline Firme & $\begin{array}{l}\text { Un IP de gran actividad (nelfinavir o ritonavir) más dos INTI (las combinaciones de dos INTI mejor } \\
\text { estudiadas en niños son zidovudina y didanosina, zidovudina y lamivudina, y estavudina y } \\
\text { didanosina). } \\
\text { En niños que puedan tomar cápsulas: el INNTI efavirenz más dos INTI, o efavirenz más el IP } \\
\text { nelfinavir y un INTI. }\end{array}$ \\
\hline Como alternativa & $\begin{array}{l}\text { Nevirapina y dos INTI. } \\
\text { Abacavir más zidovudina y lamivudina. } \\
\text { Lopinavir/ritonavir (Kaletra }{ }^{\circledR} \text { ) más dos INTI o un INTI y un INNTI. } \\
\text { Indinavir o saquinavir (cápsulas de gelatina blanda) más dos INTI en niños que puedan tragar } \\
\text { cápsulas. }\end{array}$ \\
\hline En circunstancias especiales & $\begin{array}{l}\text { Dos INTI. } \\
\text { Amprenavir más dos INTI o abacavir. }\end{array}$ \\
\hline No recomendado & $\begin{array}{l}\text { Cualquier monoterapia. } \\
\text { Estavudina y zidovudina. } \\
\text { Zalcitabina y didanosina. } \\
\text { Zalcitabina y estavudina. } \\
\text { Zalcitabina y lamivudina. }\end{array}$ \\
\hline
\end{tabular}

IP: inhibidores de la proteasa. INTI: inhibidores nucleosídicos de la transcriptasa inversa. INNTI: inhibidores no nucleosídicos de la transcriptasa inversa 
ción de dos INTI más un IP. Estos regímenes triples son más eficaces que los consistentes únicamente en dos INTI o un INTI y un IP. Otro régimen muy recomendado consiste en la combinación de efavirenz (un INNTI) con uno o dos INTI y un IP, aunque tiene la desventaja de incluir fármacos de las tres clases existentes en la actualidad, lo cual limita las opciones terapéuticas futuras en caso de fracaso del tratamiento y de que se desarrolle resistencia a las tres clases de antirretrovíricos. En adultos se han obtenido excelentes resultados con un régimen "ahorrador" de IP consistente en la combinación de efavirenz, zidovudina y lamivudina, pero no hay datos sobre este régimen en pacientes pediátricos.

Los regímenes antirretrovíricos recomendados como alternativa incluyen: nevirapina y dos INTI; tres INTI (abacavir, zidovudina y lamivudina); la formulación combinada de los IP lopinavir y ritonavir ${ }^{\circledR}$ más dos INTI o un INTI y un INNTI; las cápsulas de gelatina blanda de saquinavir más dos INTI, y el indinavir más dos INTI. Aunque todos estos regímenes han demostrado ser eficaces para suprimir la replicación del virus en algunos niños, la experiencia con ellos es todavía escasa, la duración de la supresión todavía no está bien definida y la eficacia podría no superar los potenciales riesgos de toxicidad de algunos fármacos, como el indinavir y el abacavir.

Los regímenes recomendados como tratamiento inicial solo en circunstancias especiales consisten en la combinación de dos INTI, la combinación del IP amprenavir con abacavir, y la combinación de amprenavir con dos INTI. La formulación líquida de amprenavir no se debería usar en menores de 3 años debido a su alto contenido de polietilenglicol y vitamina $\mathrm{E}$.

Los regímenes no recomendados son las monoterapias y algunas combinaciones de dos INTI, bien por su antagonismo farmacológico o por el riesgo de potenciación de sus efectos tóxicos.

\section{Problemas relacionados con la posología de los antirretrovíricos en recién nacidos}

Hay escasos datos sobre la posología de los antirretrovíricos en recién nacidos. En general, hay que tener en cuenta que las funciones hepática y renal son inmaduras en los recién nacidos, lo cual hace necesarios ajustes de las dosis. Los recién nacidos antes del término ( $<34$ semanas de gestación) tienen una función hepática todavía más inmadura que los recién nacidos a término. La filtración glomerular aumenta a cerca del doble en las primeras 4 y alcanza los valores del adulto hacia las 30 semanas de vida.

\section{El cambio del tratamiento antirretrovírico}

Cuándo cambiar el tratamiento antirretrovírico. Hay tres motivos que justifican el cambio del tratamiento antirretrovírico: 1) el fracaso del tratamiento actual, atestiguado por la progresión de la enfermedad según los parámetros clínicos, virológicos o inmunológicos; 2) la intolerancia al régimen actual o su toxicidad, y 3) la aparición de nuevos datos que demuestren que un nuevo régimen es superior al actual. En caso de fracaso del tratamiento hay que investigar el cumplimiento del mismo. La instauración de un nuevo régimen terapéutico debe acompañarse de frecuentes evaluaciones para vigilar su cumplimiento, tolerancia y eficacia virológica.

Consideraciones virológicas al cambiar el tratamiento antirretrovírico. Aunque hay escasa información sobre la respuesta de las concentraciones de ARN del VIH al tratamiento antirretrovírico en lactantes y niños pequeños, los principios virológicos generales que rigen estos tratamientos son los mismos para todos los pacientes infectados, independientemente de su edad. El objetivo ideal, la supresión de la replicación del virus, puede no ser alcanzable en todos los niños infectados por el VIH, pero aunque solo se obtenga una respuesta parcial, esta todavía puede proporcionar considerables beneficios clínicos.

Las normas establecidas para los adultos recomiendan considerar la posibilidad de cambiar el tratamiento en caso de que las concentraciones de ARN del VIH disminuyan en menos de $0,5 \log _{10}$ (tres veces) tras 4 semanas de tratamiento o en menos de $1,0 \log _{10}$ (10 veces) tras 8 semanas, o en caso de que no hayan disminuido hasta niveles indetectables tras 4 a 6 meses de tratamiento. Como los lactantes infectados durante el período perinatal suelen tener mayores cargas víricas que la mayoría de los adultos infectados, su respuesta virológica inicial puede tardar más tiempo en manifesarse $(8 \mathrm{a}$ 12 semanas) y también es frecuente que no se logre la reducción de la carga vírica hasta niveles indetectables. En los adolescentes sí pueden seguirse las mismas normas que en los adultos.

La respuesta virológica debe ser evaluada a las 4 semanas del inicio del tratamiento. Si las concentraciones basales de ARN del VIH son elevadas (> 1000000 copias $/ \mathrm{mL}$ ), la respuesta puede tardar hasta 8 a 12 semanas en manifestarse, pero si son similares a las habituales en los adultos $(<100000$ copias $/ \mathrm{mL}$ ), la respuesta debería ser detectable a las 4 semanas. Una vez que se haya logrado la máxima respuesta virológica, se deben medir las concentraciones de ARN del VIH cada 3 meses, 
para vigilar su mantenimiento. Antes de cambiar el tratamiento es necesario efectuar dos determinaciones de la carga vírica con un intervalo de al menos 1 semana entre ambas. El cambio del tratamiento puede estar indicado en los niños en las siguientes situaciones:

- Respuesta virológica inferior a la aceptable tras 8 a 12 semanas de tratamiento. En niños tratados con dos INTI y un IP, la respuesta aceptable se define como una reducción de 1,0 $\log _{10}(10$ veces), y en niños tratados con regímenes menos potentes como una reducción de $0,7 \log _{10}$ (cinco veces).

- Ausencia de supresión hasta niveles indetectables tras 4 a 6 meses de tratamiento. Sin embargo, en los niños no siempre se puede lograr este objetivo y al valorar su consecución hay que tener en cuenta las concentraciones basales. Así, por ejemplo, puede no ser necesario un cambio de tratamiento en caso de que haya una reducción sostenida de 1,5 a 2,0 $\log _{10}$, a pesar de que las concentraciones de ARN del VIH sigan siendo detectables.

- Detección repetida de ARN del VIH en niños cuyas concentraciones fueran anteriormente indetectables, lo cual sugiere problemas de cumplimiento del tratamiento o de biodisponibilidad de los fármacos.

- Un aumento confirmado del número de copias de ARN del VIH en niños en los que se haya logrado una respuesta sustancial, pero que aún sigan teniendo concentraciones detectables. Dicho aumento justificaría el cambio del tratamiento si fuera $>0,5 \log _{10}$ (tres veces) en mayores de 2 años o $>0,7 \log _{10}$ (cinco veces) en menores de 2 años.

Consideraciones inmunológicas al cambiar el tratamiento antirretrovírico. Los recuentos y porcentajes de células $\mathrm{CD}^{+}$son predictores independientes de la progresión de la enfermedad y de la mortalidad. Por cada reducción absoluta de 5 percentiles en el porcentaje de células $\mathrm{CD}^{+}$, la razón de riesgo de mortalidad aumenta en 1,3 unidades (intervalo de confianza del 95\%: 1,2 a 1,5), independientemente de la concentración de ARN del VIH. Antes de cambiar el tratamiento debido a una reducción de los linfocitos T CD4 ${ }^{+}$es necesario confirmarla con al menos una nueva determinación con un intervalo mínimo de 1 semana entre ambas. El cambio del tratamiento puede estar indicado en los niños en las siguientes situaciones:

- Cambio de la categoría inmunológica. No obstante, hay que tener en cuenta que un cambio mí- nimo de las cifras de linfocitos $\mathrm{T} \mathrm{CD}^{+}$puede modificar la categoría inmunológica (por ejemplo, una reducción del porcentaje de estas células del 26 al $24 \%$ o del 16 al $14 \%$ ) y no ser tan preocupante como un cambio rápido y sustancial de las cifras dentro de la misma categoría inmunológica.

- En los niños con porcentajes de linfocitos T CD4 ${ }^{+}$ $<15 \%$ (categoría inmunológica 3), una reducción persistente de cinco percentiles o más.

- Una reducción rápida y considerable del recuento absoluto de células T CD4 ${ }^{+}$(esto es, > 30\% en menos de 6 meses).

Consideraciones clínicas al cambiar el tratamiento antirretrovírico. La aparición de determinadas manifestaciones clínicas durante el tratamiento antirretrovírico es prueba de progresión de la enfermedad y constituye un signo de mal pronóstico. El cambio del tratamiento puede estar indicado en los niños en las siguientes situaciones:

- Deterioro progresivo del desarrollo neurológico (afectación del crecimiento cerebral, deterioro de la función cognitiva documentado por pruebas psicométricas o disfunción motora clínicamente manifiesta). En tal caso, el nuevo tratamiento debería incluir al menos un fármaco antirretrovírico con una considerable penetración en el sistema nervioso central.

- Fracaso del crecimiento.

- Progresión de la enfermedad de una categoría clínica a la siguiente. No obstante, en pacientes con parámetros inmunológicos y virológicos estables esta progresión puede no constituir una indicación para el cambio del tratamiento. Por ejemplo, la aparición de nuevas infecciones oportunistas, sobre todo en pacientes que ya tenían inmunodepresión grave al comenzar el tratamiento, puede no reflejar el fracaso del tratamiento antirretrovírico, sino la persistencia de la disfunción inmunitaria, pese a una respuesta antivírica adecuada.

La elección del nuevo régimen antirretrovírico. La elección de un nuevo régimen antirretrovírico es dictada por las indicaciones que motivaron el cambio del tratamiento y los antirretrovíricos alternativos que todavía quedan disponibles. Al elegir un nuevo tratamiento en niños que ya han recibido antirretrovíricos se deberían seguir los principios enumerados a continuación.

- Cuando el cambio haya sido motivado por la toxicidad o la intolerancia se deben elegir fármacos con diferentes perfiles de toxicidad y efectos colatera- 
les. En caso de intolerancia es posible cambiar solo un fármaco de un régimen combinado o simplemente reducir su dosis, aunque solo hasta niveles que se sepa que conservan la eficacia terapéutica.

- En caso de fracaso del tratamiento, antes de cambiarlo hay que investigar la posible participación del incumplimiento del régimen terapéutico como causa de su fracaso.

- Si el paciente ha cumplido el régimen prescrito hay que suponer que se ha creado resistencia y es aconsejable cambiar al menos dos de los fármacos. El cambio de uno solo o la adición de otro al régimen anterior no es una medida óptima. A ser posible, el nuevo régimen debería constar de al menos tres fármacos y en su elección se debería tener en cuenta la posibilidad de resistencia cruzada.

- Al cambiar de tratamiento hay que revisar las posibles interacciones farmacológicas con todas las demás medicaciones que esté tomando el paciente.

- Al cambiar de tratamiento debido a la progresión de la enfermedad en un paciente con enfermedad avanzada hay que considerar la calidad de vida.

\section{Las pruebas de resistencia a los fármacos antirretrovíricos}

La resistencia a los antirretrovíricos es importante, pero no es la única causa de fracasco del tratamiento. La obtención de una supresión vírica óptima $(<50$ copias $/ \mathrm{mL})$ reduce la probabilidad de que aparezca resistencia genotípica o fenotípica. Existen pruebas genotípicas para detectar variantes genéticas específicas (mutantes) del $\mathrm{VIH}^{2}$. Estas pruebas se basan en técnicas de amplificación y generalmente permiten detectar mutaciones en muestras de plasma con más de 1000 copias $/ \mathrm{mL}$ de ARN del VIH. En las pruebas fenotípicas se miden las concentraciones inhibidoras del $50 \%$ o del $90 \%$

2 En http://hiv-web.lanl.gov se puede encontrar un resumen de las mutaciones más frecuentes del VIH-1 seleccionadas por cada una de las tres clases de fármacos antirretrovíricos. de un determinado fármaco frente al virus. Estas pruebas proporcionan información importante acerca de la sensibilidad del virus dominante, pero pueden no detectar especies menores de virus resistentes. Las pruebas de resistencia pueden ser útiles para guiar la elección de los tratamientos iniciales y los cambios del tratamiento, pero todavía no se ha establecido su valor en los niños ni se pueden hacer recomendaciones específicas sobre su empleo para guiar las elecciones terapéuticas en este grupo. Estas pruebas se deben realizar siempre mientras todavía se estén administrando los fármacos antirretrovíricos, pues en ausencia de su presión es muy probable que los virus mutantes sean sustituidos por el virus silvestre. En la interpretación de los resultados de estas pruebas hay que tener en cuenta que la presencia de resistencia a un fármaco indica que es improbable que este suprima la replicación vírica, pero que la ausencia de resistencia no garantiza que su uso sea útil, sobre todo si tiene resistencia cruzada con otros fármacos utilizados anteriormente.

\section{SYNOPSIS}

\section{Guidelines for the use of antiretroviral agents to treat HIV infection in pediatric patients}

This document presents the most recent recommendations issued by the Task Force on the Antiretroviral and Medical Treatment of Children with HIV Infection on the management of HIV-infected children and adolescents with antiretroviral drugs. Topics discussed include the diagnosis of the infection in newborns, surveillance of pediatric HIV infection (immunologic and viral criteria), specific problems surrounding treatment with antiretroviral agents and compliance in children and adolescents, therapeutic recommendations in symptomatic and asymptomatic patients, and the use of tests for detecting resistance to antiretorviral drugs. In general, the Task Force recommends at least three antiretroviral agents combining two reverse transcriptase nucleoside inhibitors (RTNI) plus one protease inhibitor (PI) or combining efavirenz (a reverse transcriptase non-nucleoside inhibitor) plus one or two RTNI and one PI. Finally, recommendations are made on when to change an antiretroviral treatment due to lack of efficacy or toxicity or to the development of more effective treatments. 